\title{
Optimization of the dietary protein and energy levels for Dhakai quail during growing period \\ ${ }^{*}$ S. Faruque ${ }^{1}$, H. Khatun ${ }^{1}$, M.S. Islam ${ }^{1}$ and S.M.A. Rahman ${ }^{2}$
}

\author{
${ }^{1}$ Poultry Production Research Division (PPRD), Bangladesh Livestock Research Institute, Savar, \\ Dhaka 1341, Bangladesh. \\ ${ }^{2}$ Department of Animal Breeding and Genetics, Bangladesh Agricultural University, Mymensingh- \\ 2202. \\ *Corresponding author: Shakila_blri@yahoo.com
}

\begin{abstract}
A feeding trial was conducted to investigate the optimum level of dietary protein and energy on production performance and carcass characteristics of quail. A total number of 128 day-old chicks of Dhakai quail were used from internal hatchery of Bangladesh Livestock Research Institute, Savar, Dhaka. The chicks were arranged according to a $4 \times 2$ factorial (4 protein levels; 20, 22, 24 and 26\% and 2 energy levels; 2800 and $2900 \mathrm{kcal} \mathrm{ME} / \mathrm{kg}$ ), and allocated into eight dietary treatment combinations according to the experimental design. The combinations were designated as $\mathrm{D}_{1}$ $\left(\mathrm{CP}_{20} \mathrm{ME}_{2800}\right), \mathrm{D}_{2}\left(\mathrm{CP}_{20} \mathrm{ME}_{2900}\right), \mathrm{D}_{3}\left(\mathrm{CP}_{22} \mathrm{ME}_{2800}\right), \mathrm{D}_{4}\left(\mathrm{CP}_{22} \mathrm{ME}_{2900}\right), \mathrm{D}_{5}\left(\mathrm{P}_{24} \mathrm{ME}_{2800}\right), \mathrm{D}_{6}\left(\mathrm{P}_{24} \mathrm{ME}_{2900}\right), \mathrm{D}_{7}$ $\left(\mathrm{P}_{26} \mathrm{ME}_{2800}\right)$ and $\mathrm{D}_{8}\left(\mathrm{P}_{26} \mathrm{ME}_{2900}\right)$. There were 16 chicks per treatment, each treatment had 2 replications having 8 chicks in each. At the end of 5 weeks, 2 birds from each replication were randomly selected and slaughtered to analyze the meat yield traits. All birds were fed ad libitum with treatment diets from day-old to 35 days of age. There were significant effect $(\mathrm{P}<0.001)$ of crude protein levels on final body weight, total weight gain, daily gain, feed intake and feed conversion ratio (FCR). Feed intake, total weight gain and FCR at different energy levels did not differ significantly $(\mathrm{P}>0.05)$. It was observed that the performance of Dhakai quail fed diet with $22 \%$ crude protein and $2900 \mathrm{kcal}$. $\mathrm{ME} / \mathrm{kg} \mathrm{DM}$ was better compared to others in terms of total gain, daily gain, FCR and dressing percent. Futher, treatments effect of protein level was observed on weight gain from day-old to 35 days. A crude protein level of $22.227 \%$ was estimated by regression equations for growing Dhakai quail.
\end{abstract}

Keywords: Protein and energy level, Dhakai quail, Growth performance, Carcass characteristics.

\section{Introduction}

In Bangladesh, quail draws attention to the poultry farmers as it offers convenience of management. No demand of routine vaccination and medication, growing more number of birds per unit of floor space and shorter reproduction cycle with earlier marketing age offered fast monetary circulation with quicker returns. It has high prospect for reducing the protein gap of a third world country like Bangladesh. Dhakai quail may be an effective alternative to the traditional chickens and ducks for the farmers interested in poultry production in Bangladesh. Feed cost accounts for $60-70 \%$ of the total cost of poultry production, which is increasing day by day throughout the world. Therefore, emphasis must be given in formulation of economic and quality quail feed. /High quality protein with adequate amino acid balance is one of the most important prerequisite for quality diet. Protein is also one of the most expensive nutrients in the diet. Excessive protein intake results in higher nitrogen excretion and lower feed efficiency for growth and egg production. Protein provides amino acids necessary for the tissue growth and egg production. The dietary protein requirement of quail is influenced by metabolizable energy contents and the ingredient used in diet formulation. The amount of feed intake depends upon the metabolizable energy (ME) content 
of the diet, age of the bird, sex, their reproductive status, genotypes and the ambient temperature. The main energy sources usually used in diet formulation are the grains and cereals. Fat such as animal tallow, lard or other vegetable oils are added to the diet if high energy is required by the quail. However, most of the data on nutritional requirements were obtained in other countries that have different climatic conditions, preventing that an adequate feeding program is established. Thus, emphasis should be given to find out the precise information on the exact nutritional requirement of quails raised in Bangladesh.

Published reports suggest the protein (Mishra et al. 1993; Wilson et al. 1959; Woodard et al. 1973, NAS 1969, Lee et al. 1977a \& b, Hyankova et al. 1997) and energy (Farrell et al. 1982) requirements of quails in temperate regions. Further, findings under our tropic condition (Shrivastav and Panda 1982) on quail production are also available. Dhakai quail may be originated from Japanese quail (Coturnix japonica) is limited and has been introduced in Bangladesh. Unfortunately, nutritional research is somewhat limited to 'standard' quail, and a large proportion of nutritional recommendations are still often simple extrapolations from works on other poultry species. A lot more needs to be done in nutrition research and these efforts would be more productive if the different studies were part of a concerned scheme to establish Dhakai quail nutritional requirements. Considering the above-mentioned facts, the experiment was undertaken to investigate the actual requirement of crude protein and energy

for Dhakai quails in the growing periods and to know their carcass characteristics under similar management condition.

\section{Materials and Methods}

\section{Experimental design and birds}

The study was conducted during March to April, 2008 in order to investigate the optimum level of dietary protein and energy on the production performance and carcass characteristics of Dhakai quail. A feeding trial was conducted for a period of 5 weeks with 128 day-old chicks of Dhakai quail collected from internal hatchery of Bangladesh Livestock Research Institute, Savar, Dhaka. The chicks were arranged according to a $4 \times 2$ factorial (4 protein levels; 20, 22, 24 and 26\% and 2 energy levels; 2800 and $2900 \mathrm{kcal} \mathrm{ME} / \mathrm{kg}$ ), and allocated into eight dietary treatment combinations. The treatment combinations were designated as $\mathrm{D}_{1}\left(\mathrm{CP}_{20} \mathrm{ME}_{2800}\right), \quad \mathrm{D}_{2}\left(\mathrm{CP}_{20} \mathrm{ME}_{2900}\right), \quad \mathrm{D}_{3}\left(\mathrm{CP}_{22} \mathrm{ME}_{2800}\right), \quad \mathrm{D}_{4}\left(\mathrm{CP}_{22} \mathrm{ME}_{2900}\right), \quad \mathrm{D}_{5}\left(\mathrm{P}_{24} \mathrm{ME}_{2800}\right)$, $\mathrm{D}_{6}\left(\mathrm{P}_{24} \mathrm{ME}_{2900}\right), \mathrm{D}_{7}\left(\mathrm{P}_{26} \mathrm{ME}_{2800}\right)$ and $\mathrm{D}_{8}\left(\mathrm{P}_{26} \mathrm{ME}_{2900}\right)$. There were 16 chicks per treatment combination. Each treatment combination had 2 replications.

\section{Formulation of diets}

The ingredient used to prepare diet were maize, wheat, rice polish, soya bean meal, protein concentrates, limestone (calcium carbonate) and synthetic methionine and lysine. The ingredients were purchased from the local feed company. A premix containing trace minerals and vitamins was mixed with all diets. Further, common salt and DCP was added to the diets as per requirements. The chicks under the experiment were fed mash feed ad libitum during the entire trial period.

\section{Recording performance data}

Feeding trial was conducted for a period of 5 weeks. Initial body weight of day old chicks was recorded immediately before the start of the trial and then weekly thereafter. All birds were weighed individually, before supplying morning feed and water. The feed residues were collected and weighed on the following morning to calculate the actual feed intakes. Feed intake including left over was recorded daily and was calculated from the difference between 
the feed supplied and it's left over. Feed conversion ratio (FCR) was recorded for the whole period as total feed intake $(\mathrm{g})$ per weight gain $(\mathrm{g})$. Temperature and humidity $(\%)$ were recorded three times a day; in the morning $(0900 \mathrm{~h})$, noon $(1300 \mathrm{~h})$ and evening $(1700 \mathrm{~h})$. Health status of birds was monitored everyday and mortality of birds was recorded, if any.

\section{Slaughtering, morphological and carcass characteristics}

At 5 weeks of age, two birds from each treatment (out of 8) were slaughtered to analyze the meat yield traits. All birds to be slaughtered were kept off feed overnight but drinking water was provided ad libitum at all times. Birds were slaughtered following 'halal' method (Singh et al., 2003) by severing the jugular vein and allowed to bleed completely and then plucked and weighed to determine blood and feather losses (Kotula et al., 1960; Pandey and Shyamsunder, 1990). Data on pre-slaughter live weight, blood loss weight, eviscerated weight, shank length and weight, neck length and weight, head weight, breast meat weight, drumstick weight and length, thigh bone length and weight, digestive tract weight, gizzard weight, liver weight, spleen weight, heart weight, back and breast bone weight, wing bone length and weight, Giblet and skin weight were recorded. Morphological characteristics such as length of neck, thigh, drumstick, shank and wing were recorded by a measuring scale.

\section{Statistical analysis}

All data were analyzed by $4 \times 2$ factorial (Snedecor and Cochran, 1989) using the procedure of SPSS 10 for Windows (SPSS, 1998). Means were compared for the significant differences using Duncans Multiple Range Test (Duncan, 1955). Correlation and regression analyses were done according to Snedecor and Cochran (1989).

\section{Results and Discussion}

\section{Effects of protein on the production performance}

The weight of day-old quail chicks did not differ significantly $(\mathrm{P}>0.05)$ among the dietary crude protein levels (Table1). There were significant effect $(\mathrm{P}<0.001)$ of crude protein levels on final body weight, total weight gain, daily gain feed intake and feed conversion ratio. Total weight gain was significantly $(\mathrm{P}<0.001)$ higher in $22 \%$ crude protein level $(94.17 \mathrm{~g})$, but there was no difference in total weight gain between $20 \%(83.96 \mathrm{~g}), 24 \%(88.06 \mathrm{~g})$ and $26 \%(88.35 \mathrm{~g})$ crude protein levels. It might be due to the efficient utilization of feed containing $22 \%$ crude protein level by the birds than that of others levels of crude proteins. Several factors might contribute to the differences in adult body weight, weight of day-old chicks, egg weight and differences in management of the stock. Significantly $(\mathrm{P}<0.001)$ higher daily weight gain was recorded in $22 \%$ crude protein level $(2.46 \mathrm{~g})$, but there was no differences between 20\% (2.16 g) , 24\% (2.28 g) and 26\% (2.29 g) crude protein levels. There was highly significant $(\mathrm{P}<0.001)$ effect of crude protein levels on feed intake and feed conversion ratio. Although final body weight of Dhakai quail was the highest with levels of $22 \%$ crude protein. In this trial, there was a quadratic effect $(\mathrm{P}=0.36)$ of crude protein levels on total weight gain and a level of $22.227 \%$ was estimated by regression analysis for maximum gain.

In the present study, estimated protein levels were lower than the levels reported by other studies with quails at similar age. Lepore and Marks (1971) reported heavier quails at four weeks of age, when fed diets with $25 \%$ crude protein. Lee et al. (1977a) evaluated protein requirements of Japanese quails in the growing period and reported better growth performance with levels of $24 \%$ to $32 \%$ CP. Shim \& Vohra (1984) recommended $24 \%$ CP in diets for growing Japanese quails, which could be lowered to $20 \%$ after three weeks of age. 
Table 1. Production performance of Dhakai quails up to 35 days of age according to the crude protein level of the diet

\begin{tabular}{ccccccc}
\hline $\begin{array}{c}\text { Crude Protein } \\
(\%)\end{array}$ & $\begin{array}{c}\text { Initial } \\
\text { Weight } \\
(\mathrm{g})\end{array}$ & $\begin{array}{c}\text { Final } \\
\text { Weight } \\
(\mathrm{g})\end{array}$ & $\begin{array}{c}\text { Total } \\
\text { Gain } \\
(\mathrm{g} / \mathrm{b})\end{array}$ & $\begin{array}{c}\text { Daily } \\
\text { Gain } \\
(\mathrm{g} / \mathrm{b} / \mathrm{d})\end{array}$ & $\begin{array}{c}\text { Feed } \\
\text { Intake }^{2} \\
(\mathrm{~g})\end{array}$ & $\begin{array}{c}\text { Feed Conversion } \\
(\mathrm{g} / \mathrm{g})\end{array}$ \\
\hline 20 & 8.06 & $83.96^{\mathrm{b}}$ & $75.87^{\mathrm{b}}$ & $2.16^{\mathrm{b}}$ & $406.31^{\mathrm{b}}$ & $5.36^{\mathrm{b}}$ \\
22 & 7.96 & $94.17^{\mathrm{a}}$ & $86.27 \mathrm{a}$ & $2.46^{\mathrm{a}}$ & $405.28^{\mathrm{b}}$ & $4.68^{\mathrm{a}}$ \\
24 & 8.31 & $88.06^{\mathrm{b}}$ & $79.80^{\mathrm{b}}$ & $2.28^{\mathrm{b}}$ & $415.83^{\mathrm{b}}$ & $5.21^{\mathrm{b}}$ \\
26 & 8.15 & $88.35^{\mathrm{b}}$ & $80.16^{\mathrm{b}}$ & $2.29^{\mathrm{b}}$ & $431.26^{\mathrm{a}}$ & $5.40^{\mathrm{b}}$ \\
SED & 0.089 & 0.84 & 0.85 & 0.024 & 1.887 & 0.041 \\
F value and & $0.677^{\mathrm{NS}}$ & $6.104^{* * * *}$ & $6.293^{* * *}$ & $6.249^{* * * *}$ & $10.175^{* * *}$ & $16.737^{* * * *}$ \\
significance & & & & & & \\
\hline
\end{tabular}

Means having different superscripts in the same row differ significantly $(\mathrm{P}<0.001)$

$\mathrm{FCR}=$ Feed conversion ratio, $\mathrm{g}$ feed $/ \mathrm{g}$ gain; $\mathrm{NS}=$ Non-significance $(\mathrm{p}>0.05)$.

1-Quadratic effect $(\mathrm{p}=0.36) \mathrm{y}=-255.65+29.185 \mathrm{x}-0.6275 \mathrm{x}^{2}, \mathrm{r} 2=0.70$

2- Quadratic effect $(\mathrm{p}=0.08) \mathrm{y}=855.53-43.053 \mathrm{x}+1.0288 \mathrm{x}^{2}, \mathrm{r}^{2}=0.83$

\section{Effect of energy on the production performance}

Body weight gain, feed intake and FCR of Dhakai quails at two energy levels are presented in Table 2. Feed intake, total weight gain and FCR at different energy levels did not differ significantly $(\mathrm{P}>0.05)$. The amount of feed intake depends on the metabolizable energy (ME) contents of the diet, age of the birds, their reproductive status and the ambient temperature. An energy requirement of 2,600 to 3,000 kcal.ME $/ \mathrm{kg}$ diet for growing quail has been reported from temperate regions (Farrell et al., 1982). Though raising the dietary energy levels from 2,600 to $2800 \mathrm{kcalME} / \mathrm{kg}$ did not influence the gain in weight, it affected significantly the efficiency of feed utilization as the feed consumption was reduced significantly (Shrivastav and Panda, 1982).

Table 2. Production performance of Dhakai quails up to 35 days of age according to the energy level of the diet.

\begin{tabular}{l|cc|c|c}
\hline \multirow{2}{*}{ Parameter } & \multicolumn{2}{|c|}{ Energy (kcal/kg DM) } & SED & $\begin{array}{c}\text { F value and the level } \\
\text { of significance }\end{array}$ \\
\cline { 2 - 3 } & 2800 & 2900 & & $0.015^{\mathrm{NS}}$ \\
Body weight gain (g) & 80.55 & 80.32 & 0.908 & $0.500^{\mathrm{NS}}$ \\
Feed intake (g) & 412.67 & 416.67 & 2.827 & $0.336^{\mathrm{NS}}$ \\
FCR & 5.12 & 5.20 & 0.072 & \\
\hline
\end{tabular}

NS=Non-significance $(\mathrm{p}>0.05)$

There was an interaction between protein and energy levels on growth performance of Dhakai quails (Table 3). Although final body weight, total weight gain and daily gain were the highest with the combination of $22 \% \mathrm{CP}$ and $2900 \mathrm{kcalME} / \mathrm{kg}$ based diet, the interaction effects between protein and energy levels on growth performances were not significant ( $(\mathrm{P}>0.05)$. From Table 4, it was seen that $22 \% \mathrm{CP}$ and $2900 \mathrm{kcalME} / \mathrm{kg}$ based diet decreased feed intake but increased final body weight, total gain and daily gain.

Table 3. Interaction of different protein and energy levels on growth performance of growing quails

\begin{tabular}{|c|c|c|c|c|c|c|c|}
\hline \multirow[t]{2}{*}{ Parameter } & \multirow{2}{*}{$\begin{array}{c}\text { Dietary } \\
\text { ME level } \\
\text { (kcal/kg) }\end{array}$} & \multicolumn{4}{|c|}{ Dietary CP level (\%) } & \multirow[t]{2}{*}{ Mean } & \multirow[t]{2}{*}{$\mathrm{CP} \times \mathrm{ME}$} \\
\hline & & 20 & 22 & 24 & 26 & & \\
\hline $\begin{array}{l}\text { Day old } \\
\text { weight }(\mathrm{g})\end{array}$ & $\begin{array}{l}2800 \\
2900\end{array}$ & $\begin{array}{l}8.0 \\
7.8\end{array}$ & $\begin{array}{l}8.06 \\
8.12\end{array}$ & $\begin{array}{l}8.33 \\
8.20\end{array}$ & $\begin{array}{l}8.31 \\
8.06\end{array}$ & $\begin{array}{l}8.18 \\
8.04\end{array}$ & $0.131^{\mathrm{NS}}$ \\
\hline
\end{tabular}




\begin{tabular}{|l|c|c|c|c|c|c|c|}
\hline & Mean & 7.8 & 8.09 & 8.26 & 8.19 & 8.11 & \\
\hline Final body & 2800 & 81.8 & 93.85 & 91.20 & 88.43 & 88.73 & $1.680^{\mathrm{NS}}$ \\
weight (g) & 2900 & 86.0 & 94.46 & 84.93 & 88.26 & 88.37 & \\
& Mean & 83.96 & 94.17 & 88.06 & 88.35 & 88.55 & \\
\hline Total weight & 2800 & 73.73 & 85.85 & 82.86 & 80.12 & 80.55 & $1.606^{\mathrm{NS}}$ \\
gain (g) & 2900 & 77.87 & 86.66 & 76.73 & 80.20 & 80.32 & \\
& Mean & 75.87 & 86.26 & 79.80 & 80.16 & 80.43 & \\
\hline Gain (g/d/b) & 2800 & 2.10 & 2.45 & 2.36 & 2.28 & 2.30 & $1.614^{\mathrm{NS}}$ \\
& 2900 & 2.22 & 2.47 & 2.19 & 2.29 & 2.29 & \\
& Mean & 2.16 & 2.46 & 2.28 & 2.29 & 2.29 & \\
\hline Total feed & 2800 & 398.7 & 401.2 & 418.4 & 432.3 & 412.6 & $1.738^{\mathrm{NS}}$ \\
intake $(\mathrm{g})$ & 2900 & 413.9 & 409.3 & 413.2 & 430.2 & 416.6 & \\
& Mean & 406.3 & 405.2 & 415.8 & 431.2 & 414.6 & \\
\hline FCR & 2800 & 5.40 & 4.66 & 5.03 & 5.40 & 5.12 & $1.548^{\mathrm{NS}}$ \\
& 2900 & 5.33 & 4.70 & 5.40 & 5.40 & 5.20 & \\
& Mean & 5.36 & 4.68 & 5.21 & 5.40 & 5.16 & \\
\hline * $<0.05 ; * *$ P $<0.01 ; * * *$ P $<0.001 ;$ NS=Non-significance (p $>0.05) ; \mathrm{FCR}=$ Feed conversion ratio & & \\
\end{tabular}

\section{Morphological characteristics}

Effects of different levels of protein and energy on morphological characteristics of Dhakai quail are presented in Table 4. Although interaction of different levels of protein and energy based diet had no effect on neck length, thigh length, shank length and wing bone length, it affected on the length of drumstick $(\mathrm{P}<0.001)$. Different levels of $\mathrm{CP}$ had no effect on morphological characteristics but different levels of energy had some effect on neck length $(\mathrm{P}<0.001)$ and shank length $(\mathrm{P}<0.05)$.

Table 4. Morphological characteristics of Dhakai quail fed on different treatment diet

\begin{tabular}{|c|c|c|c|c|c|c|c|c|c|}
\hline \multirow[t]{2}{*}{ Parameter } & \multirow{2}{*}{$\begin{array}{c}\text { Dietary } \\
\text { ME level } \\
\text { (kcal/kg) }\end{array}$} & \multicolumn{4}{|c|}{ Dietary CP level (\%) } & \multirow[t]{2}{*}{ Mean } & \multicolumn{3}{|c|}{ F value \& level of significance } \\
\hline & & 20 & 22 & 24 & 26 & & $\mathrm{CP}$ & ME & $\begin{array}{l}\mathrm{CP} \times \\
\mathrm{ME}\end{array}$ \\
\hline \multirow{3}{*}{$\begin{array}{l}\text { Neck } \\
\text { length }(\mathrm{cm})\end{array}$} & 2800 & 4.50 & 4.63 & 4.75 & 4.75 & 4.66 & \multirow[t]{3}{*}{$1.675^{\mathrm{NS}}$} & \multirow[t]{3}{*}{$24.405^{* * * *}$} & \multirow[t]{3}{*}{$1.504^{\mathrm{NS}}$} \\
\hline & 2900 & 4.12 & 4.38 & 4.00 & 4.38 & 4.22 & & & \\
\hline & Mean & 4.31 & 4.50 & 4.38 & 4.57 & 4.44 & & & \\
\hline \multirow{3}{*}{$\begin{array}{l}\text { Thigh } \\
\text { length }(\mathrm{cm})\end{array}$} & 2800 & 4.38 & 4.38 & 4.63 & 4.00 & 4.35 & \multirow[t]{3}{*}{$1.553^{\mathrm{NS}}$} & \multirow[t]{3}{*}{$0.088^{\mathrm{NS}}$} & \multirow[t]{3}{*}{$2.997^{\mathrm{NS}}$} \\
\hline & 2900 & 4.50 & 4.13 & 4.38 & 4.50 & 4.38 & & & \\
\hline & Mean & 4.44 & 4.25 & 4.50 & 4.25 & 4.36 & & & \\
\hline \multirow{3}{*}{$\begin{array}{l}\text { Drumstick } \\
\text { length }(\mathrm{cm})\end{array}$} & 2800 & 3.25 & 3.25 & 3.38 & 3.50 & 3.34 & \multirow[t]{3}{*}{$1.500^{\mathrm{NS}}$} & \multirow[t]{3}{*}{$0.001^{\mathrm{NS}}$} & \multirow[t]{3}{*}{$5.972^{* *}$} \\
\hline & 2900 & 3.38 & 3.38 & 3.50 & 3.13 & 3.35 & & & \\
\hline & Mean & 3.31 & 3.31 & 3.44 & 3.31 & 3.34 & & & \\
\hline \multirow{3}{*}{$\begin{array}{l}\text { Shank } \\
\text { length }(\mathrm{cm})\end{array}$} & 2800 & 3.00 & 3.00 & 3.13 & 3.12 & 3.06 & \multirow[t]{3}{*}{$0.800^{\mathrm{NS}}$} & \multirow[t]{3}{*}{$4.863^{*}$} & \multirow[t]{3}{*}{$2.421^{\mathrm{NS}}$} \\
\hline & 2900 & 3.13 & 3.38 & 3.13 & 3.12 & 3.19 & & & \\
\hline & Mean & 3.06 & 3.19 & 3.13 & 3.12 & 3.13 & & & \\
\hline \multirow{3}{*}{$\begin{array}{l}\text { Wing bone } \\
\text { length }(\mathrm{cm})\end{array}$} & 2800 & 8.63 & 8.63 & 8.87 & 8.25 & 8.59 & \multirow[t]{3}{*}{$2.997^{\mathrm{NS}}$} & \multirow[t]{3}{*}{$0.825^{\mathrm{NS}}$} & \multirow[t]{3}{*}{$1.553^{\mathrm{NS}}$} \\
\hline & 2900 & 8.75 & 8.38 & 8.50 & 8.38 & 8.50 & & & \\
\hline & Mean & 8.69 & 8.50 & 8.68 & 8.31 & 5.55 & & & \\
\hline
\end{tabular}

$* \mathrm{P}<0.05 ; * * \mathrm{P}<0.01 ; * * * \mathrm{P}<0.001 ; \mathrm{NS}=$ Non-significance

Chemical composition of Dhakai quail meat was presented in Table 5. The data indicated that Dhakai quail meat contained more protein but less total lipid and cholesterol. The amount of protein, total lipid and cholesterol was determined at the end of the experiment was found out 
to be $67.70 \%, 8.0 \%$ and $74 \mathrm{mg} / 100 \mathrm{~g}$, respectively. El- Dengawy and Nassar (2001) reported that protein contents of thigh meat of wild male and female quails were 65.6 and $68.8 \%$, which is more or less similar to the results obtained in present study.

Table 5. Chemical composition of Dhakai quail meat

\begin{tabular}{|c|c|c|}
\hline Cholesterol $(\mathrm{mg} / 100 \mathrm{~g})$ & Total lipid $(\%)$ & Crude Protein $(\%)$ \\
\hline 74 & 8.0 & 67.70 \\
\hline
\end{tabular}

\section{Carcass characteristics}

Carcass characteristics of Dhakai quail fed on different levels of protein and energy based diets are presented in Table 6. Blood weight, feather weight and abdominal fat weight were not affected $(\mathrm{P}>0.05)$ by dietary $\mathrm{CP}$ levels but other parameters were affected significantly. There was significant effect of different ME levels on dressing percent, blood weight, feather weight, heart weight, gizzard weight and digestive tract weight. The interaction effect of different CP level x ME level on blood weight, feather weight, head weight, shank weight, wing meat weight and digestive tract weight was found no significant $(\mathrm{P}>0.05)$. On an average the quails on diet containing $22 \% \mathrm{CP}$ and $2800 \mathrm{kcal} \mathrm{ME} / \mathrm{kg}$ gained the highest live weight at slaughter i.e. 93.75 g. Dressing percentage, head weight, thigh plus drumstick weight and back plus breast bone weight of quails fed on diet containing 22\% CP and 2900 $\mathrm{kcal} \mathrm{ME} / \mathrm{kg}$ were the highest i.e. $64.35 \%, 4.62 \mathrm{~g}, 14.0 \mathrm{~g}$ and $15.62 \mathrm{~g}$; respectively. The data showed that average carcass weights expressed as a proportion of live body weight was 61.55 $\%$ for Dhakai quails, while El- Dengawy and Nassar (2001) found that the percentage of carcass weight of live weight were 76.5 and $75.3 \%$ for male and female, respectively.

Table 6. Carcass characteristics of Dhakai quail fed on different treatment diet

\begin{tabular}{|c|c|c|c|c|c|c|c|c|c|}
\hline \multirow[t]{2}{*}{ Parameter } & \multirow{2}{*}{$\begin{array}{l}\text { Dietary } \\
\text { ME level } \\
\text { (kcal/kg) }\end{array}$} & \multicolumn{4}{|c|}{ Dietary CP level (\%) } & \multirow[t]{2}{*}{ Mean } & \multicolumn{3}{|c|}{ F value \& level of significance } \\
\hline & & 20 & 22 & 24 & 26 & & $\mathrm{CP}$ & ME & $\mathrm{CP} \times \mathrm{ME}$ \\
\hline $\begin{array}{l}\text { Live weight } \\
\text { (g) }\end{array}$ & $\begin{array}{l}2800 \\
2900 \\
\text { Mean } \\
\end{array}$ & $\begin{array}{l}81.00 \\
88.50 \\
84.75\end{array}$ & $\begin{array}{l}93.75 \\
92.87 \\
93.31 \\
\end{array}$ & $\begin{array}{l}90.00 \\
77.25 \\
83.62 \\
\end{array}$ & $\begin{array}{l}91.87 \\
89.12 \\
90.50 \\
\end{array}$ & $\begin{array}{l}89.15 \\
86.93 \\
88.04\end{array}$ & $5.986^{* *}$ & $1.377^{\mathrm{NS}}$ & $4.837^{*}$ \\
\hline Dressing $(\%)$ & $\begin{array}{l}2800 \\
2900 \\
\text { Mean }\end{array}$ & $\begin{array}{l}64.23 \\
59.62 \\
61.93\end{array}$ & $\begin{array}{l}61.07 \\
64.35 \\
62.71\end{array}$ & $\begin{array}{l}62.75 \\
60.51 \\
61.63\end{array}$ & $\begin{array}{l}60.41 \\
59.49 \\
59.95\end{array}$ & $\begin{array}{l}62.11 \\
60.99 \\
61.55\end{array}$ & $8.140^{* * *}$ & $7.622^{*}$ & $16.553^{* * *}$ \\
\hline $\begin{array}{l}\text { Blood } \\
\text { weight } \\
\text { (g) }\end{array}$ & $\begin{array}{l}2800 \\
2900 \\
\text { Mean }\end{array}$ & $\begin{array}{l}3.00 \\
3.75 \\
3.37\end{array}$ & $\begin{array}{l}1.75 \\
3.37 \\
2.56\end{array}$ & $\begin{array}{l}3.25 \\
3.37 \\
3.31\end{array}$ & $\begin{array}{l}3.00 \\
4.75 \\
3.87\end{array}$ & $\begin{array}{l}2.75 \\
3.81 \\
3.28\end{array}$ & $1.493^{\mathrm{NS}}$ & $5.770^{*}$ & $0.750^{\mathrm{NS}}$ \\
\hline $\begin{array}{l}\text { Feather } \\
\text { weight } \\
\text { (g) }\end{array}$ & $\begin{array}{l}2800 \\
2900 \\
\text { Mean }\end{array}$ & $\begin{array}{l}8.50 \\
5.87 \\
7.18\end{array}$ & $\begin{array}{l}8.37 \\
5.37 \\
6.87\end{array}$ & $\begin{array}{l}5.75 \\
5.25 \\
5.50\end{array}$ & $\begin{array}{l}7.87 \\
5.50 \\
6.68\end{array}$ & $\begin{array}{l}7.62 \\
5.50 \\
6.56\end{array}$ & $2.062^{\mathrm{NS}}$ & $17.084^{* * *}$ & $1.172^{\mathrm{NS}}$ \\
\hline $\begin{array}{l}\text { Head weight } \\
\text { (g) }\end{array}$ & $\begin{array}{l}2800 \\
2900 \\
\text { Mean }\end{array}$ & $\begin{array}{l}4.12 \\
4.00 \\
4.06\end{array}$ & $\begin{array}{l}4.50 \\
4.62 \\
4.56\end{array}$ & $\begin{array}{l}5.00 \\
4.75 \\
4.87\end{array}$ & $\begin{array}{l}4.50 \\
5.00 \\
4.75\end{array}$ & $\begin{array}{l}4.53 \\
4.59 \\
4.56\end{array}$ & $8.503^{* * *}$ & $0.261^{\mathrm{NS}}$ & $1.831^{\mathrm{NS}}$ \\
\hline $\begin{array}{l}\text { Neck weight } \\
\text { (g) }\end{array}$ & $\begin{array}{c}2800 \\
2900 \\
\text { Mean }\end{array}$ & $\begin{array}{l}2.00 \\
2.12 \\
2.06\end{array}$ & $\begin{array}{l}2.37 \\
2.87 \\
2.62\end{array}$ & $\begin{array}{l}2.25 \\
2.87 \\
2.06\end{array}$ & $\begin{array}{l}2.37 \\
3.25 \\
2.81\end{array}$ & $\begin{array}{l}2.25 \\
2.53 \\
2.39\end{array}$ & $7.531^{* *}$ & $3.995^{\mathrm{NS}}$ & $3.570^{*}$ \\
\hline $\begin{array}{l}\text { Heart weight } \\
\text { (g) }\end{array}$ & $\begin{array}{c}2800 \\
2900 \\
\text { Mean }\end{array}$ & $\begin{array}{l}0.57 \\
0.56 \\
0.56\end{array}$ & $\begin{array}{l}0.77 \\
0.74 \\
0.75\end{array}$ & $\begin{array}{l}0.73 \\
0.55 \\
0.64\end{array}$ & $\begin{array}{l}0.67 \\
0.62 \\
0.65\end{array}$ & $\begin{array}{l}0.69 \\
0.61 \\
0.65\end{array}$ & $28.807^{* * * *}$ & $23.534^{* * *}$ & $6.431^{* *}$ \\
\hline
\end{tabular}




\begin{tabular}{|c|c|c|c|c|c|c|c|c|c|}
\hline $\begin{array}{l}\text { Liver weight } \\
\text { (g) }\end{array}$ & $\begin{array}{l}2800 \\
2900 \\
\text { Mean }\end{array}$ & $\begin{array}{l}1.75 \\
2.12 \\
1.93\end{array}$ & $\begin{array}{l}2.50 \\
1.87 \\
2.18\end{array}$ & $\begin{array}{l}2.00 \\
2.00 \\
2.00\end{array}$ & $\begin{array}{l}2.37 \\
2.75 \\
2.56\end{array}$ & $\begin{array}{l}2.15 \\
2.18 \\
2.17\end{array}$ & $6.951^{* *}$ & $0.088^{\mathrm{NS}}$ & $4.870^{*}$ \\
\hline $\begin{array}{l}\text { Breast meat } \\
\text { weight } \\
\text { (g) }\end{array}$ & $\begin{array}{l}2800 \\
2900 \\
\text { Mean }\end{array}$ & $\begin{array}{l}17.15 \\
19.25 \\
18.18\end{array}$ & $\begin{array}{l}20.87 \\
20.37 \\
20.62\end{array}$ & $\begin{array}{l}20.00 \\
16.37 \\
18.18\end{array}$ & $\begin{array}{l}19.25 \\
18.12 \\
18.68\end{array}$ & $\begin{array}{l}19.31 \\
18.53 \\
18.92\end{array}$ & $6.409^{* *}$ & $2.904^{\mathrm{NS}}$ & $6.632^{* *}$ \\
\hline $\begin{array}{l}\text { (Thigh + } \\
\text { Drumstick) } \\
\text { weight }(\mathrm{g})\end{array}$ & $\begin{array}{l}2800 \\
2900 \\
\text { Mean }\end{array}$ & $\begin{array}{l}11.62 \\
12.87 \\
12.25\end{array}$ & $\begin{array}{l}13.12 \\
14.00 \\
13.56\end{array}$ & $\begin{array}{l}13.25 \\
11.62 \\
12.43\end{array}$ & $\begin{array}{l}13.37 \\
12.62 \\
13.00\end{array}$ & $\begin{array}{l}12.84 \\
12.78 \\
12.81\end{array}$ & $6.349^{* * *}$ & $0.071^{\mathrm{NS}}$ & $8.293^{* * * *}$ \\
\hline $\begin{array}{l}\text { Shank } \\
\text { weight } \\
\text { (g) }\end{array}$ & $\begin{array}{l}2800 \\
2900 \\
\text { Mean }\end{array}$ & $\begin{array}{l}1.87 \\
2.00 \\
1.93\end{array}$ & $\begin{array}{l}2.37 \\
2.75 \\
2.56\end{array}$ & $\begin{array}{l}2.25 \\
2.00 \\
2.12\end{array}$ & $\begin{array}{l}2.50 \\
2.87 \\
2.68\end{array}$ & $\begin{array}{l}2.25 \\
2.40 \\
2.32\end{array}$ & $14.345^{* * *}$ & $2.763^{\mathrm{NS}}$ & $2.482^{\mathrm{NS}}$ \\
\hline $\begin{array}{l}\text { Wing meat } \\
\text { weight } \\
\text { (g) }\end{array}$ & $\begin{array}{c}2800 \\
2900 \\
\text { Mean }\end{array}$ & $\begin{array}{l}4.62 \\
4.75 \\
4.68\end{array}$ & $\begin{array}{l}5.25 \\
5.25 \\
5.25\end{array}$ & $\begin{array}{l}4.75 \\
4.87 \\
4.81\end{array}$ & $\begin{array}{l}5.62 \\
5.25 \\
5.43\end{array}$ & $\begin{array}{l}5.66 \\
5.03 \\
5.04\end{array}$ & $3.754^{*}$ & $0.030^{\mathrm{NS}}$ & $0.420^{\mathrm{NS}}$ \\
\hline $\begin{array}{l}\text { Gizzard } \\
\text { weight } \\
\text { (g) }\end{array}$ & $\begin{array}{l}2800 \\
2900 \\
\text { Mean }\end{array}$ & $\begin{array}{l}3.62 \\
2.87 \\
3.25\end{array}$ & $\begin{array}{l}3.75 \\
3.00 \\
3.37\end{array}$ & $\begin{array}{l}3.12 \\
2.12 \\
2.62\end{array}$ & $\begin{array}{l}2.75 \\
3.62 \\
3.18 \\
\end{array}$ & $\begin{array}{l}3.31 \\
2.90 \\
3.11\end{array}$ & $4.389^{*}$ & $6.571^{*}$ & $7.430^{* *}$ \\
\hline $\begin{array}{l}\text { Skin weight } \\
\text { (g) }\end{array}$ & $\begin{array}{c}2800 \\
2900 \\
\text { Mean }\end{array}$ & $\begin{array}{l}3.75 \\
5.62 \\
4.68\end{array}$ & $\begin{array}{l}6.50 \\
5.62 \\
6.06\end{array}$ & $\begin{array}{l}4.62 \\
4.62 \\
4.62\end{array}$ & $\begin{array}{l}5.62 \\
5.62 \\
5.62\end{array}$ & $\begin{array}{l}5.12 \\
5.37 \\
5.25\end{array}$ & $10.572^{* * * *}$ & $1.324^{\mathrm{NS}}$ & $7.071^{* * *}$ \\
\hline $\begin{array}{l}\text { Abdominal } \\
\text { fat weight } \\
\text { (g) }\end{array}$ & $\begin{array}{c}2800 \\
2900 \\
\text { Mean }\end{array}$ & $\begin{array}{l}0.37 \\
0.25 \\
0.31\end{array}$ & $\begin{array}{l}0.27 \\
0.40 \\
0.33\end{array}$ & $\begin{array}{l}0.27 \\
0.30 \\
0.28\end{array}$ & $\begin{array}{l}0.27 \\
0.42 \\
0.35\end{array}$ & $\begin{array}{l}0.30 \\
0.34 \\
0.32\end{array}$ & $0.781^{\mathrm{NS}}$ & $1.800^{\mathrm{NS}}$ & $3.909^{*}$ \\
\hline $\begin{array}{l}\text { Digestive } \\
\text { tract weight } \\
\text { (g) }\end{array}$ & $\begin{array}{l}2800 \\
2900 \\
\text { Mean }\end{array}$ & $\begin{array}{l}4.12 \\
3.62 \\
3.87\end{array}$ & $\begin{array}{l}5.25 \\
4.50 \\
4.87\end{array}$ & $\begin{array}{l}4.62 \\
3.50 \\
4.06\end{array}$ & $\begin{array}{l}4.62 \\
5.00 \\
4.81\end{array}$ & $\begin{array}{l}4.65 \\
4.15 \\
4.40\end{array}$ & $5.014^{*}$ & $4.816^{*}$ & $1.949^{\mathrm{NS}}$ \\
\hline $\begin{array}{l}\text { Lungs } \\
\text { weight } \\
\text { (g) }\end{array}$ & $\begin{array}{c}2800 \\
2900 \\
\text { Mean }\end{array}$ & $\begin{array}{l}0.42 \\
0.42 \\
0.42\end{array}$ & $\begin{array}{l}0.42 \\
0.42 \\
0.42\end{array}$ & $\begin{array}{l}0.45 \\
0.43 \\
0.44\end{array}$ & $\begin{array}{l}0.42 \\
0.44 \\
0.43\end{array}$ & $\begin{array}{l}0.43 \\
0.43 \\
0.43\end{array}$ & $7.833^{* *}$ & $0.001^{\mathrm{NS}}$ & $9.833^{* * * *}$ \\
\hline $\begin{array}{l}\text { Back } \\
\text { bone+Breast } \\
\text { bone weight } \\
\text { (g) }\end{array}$ & $\begin{array}{l}2800 \\
2900 \\
\text { Mean }\end{array}$ & $\begin{array}{c}9.50 \\
12.00 \\
10.75\end{array}$ & $\begin{array}{l}12.75 \\
15.62 \\
14.18\end{array}$ & $\begin{array}{l}13.37 \\
10.25 \\
11.18\end{array}$ & $\begin{array}{l}12.37 \\
11.87 \\
12.12\end{array}$ & $\begin{array}{l}12.00 \\
12.43 \\
12.21\end{array}$ & $6.185^{* * *}$ & $0.572^{\mathrm{NS}}$ & $5.923^{* * *}$ \\
\hline
\end{tabular}

$* \mathrm{P}<0.05 ; * * \mathrm{P}<0.01 ; * * * \mathrm{P}<0.001 ; \mathrm{NS}=$ Non-significance

\section{Conclusion}

Based on the present findings it may be concluded that the performance of Dhakai quail fed on dietary crude protein level of $22 \%$ and $2900 \mathrm{kcal}$. ME/kg DM was better compared to other counterparts in terms of total gain, daily gain and dressing \%. The findings of this study suggested that the levels of $22 \%$ of crude protein estimated by regression analysis, in the diet of Dhakai quails are recommended for the growing period (0 to 35 days) for better growth. For better perfection of energy level, another nutritional study is required.

\section{References}

Duncan, D.B. 1955. Multiple Range and Multiple F-tests. Biometrics 11:1-42.

El-Dengawy, R.A. and Nassar, A.M. 2001. Investigation on the nutritive value and microbiological quality of wild quail carcasses. Nahrung/Food 45. No. 1, pp. 50-54. 
Farrell, D.J., Atmamihardia, S.I. and Pym, P.A.E. 1982. Calorimetric measurement of the energy and nitrogen metabolism of Japanese quail. Farrell, D.J., S. I. Atmamihardia, and P. A. E. Pym. 1982. Calorimetric measurement of the energy and nitrogen metabolism of Japanese quail. Br. Poultry Sci., 23: 375-382.

Hyankova L., Dedkova, L., Knizetva, H. and Klecner, D. 1997. Responses in growth, food intake and food conversion efficiency to different dietary protein concentrations in meat-type lines of Japanese quail. Br. Poultry Sci., 38(5):564-570.

Kotula A.W., Thomson, J.E. and Kinner, J.A. 1960. Weight increase during chilling of broilers as influenced by methods of opening the abdominal cavity during evisceration. Poult. Sci., 39: 26-27.

Lee T.K., Shim, K.F. and Tan, E.L. 1977a. Protein requirement of growing Japanese quails in the tropics.Sing. J. Pri. Ind., 5: 70-81.

Lee T.K., Shim, K.F. and Tan, E.L. 1977b. Protein requirement of laying Japanese quails in the tropics. Sing. J. Pri. Ind., 5: 82-90.

Lepore, R.D. and Marks, H.L. 1971. Growth rate inheritance in Japanese quail. Protein and energy requirements of lines selected under different nutritional environments. Poult. Sci., 50:1335-1341

Mishra, S.K., Panda, B., Mohapatra, S.C. and Ahuja, S.D. 1993. Genotype-protein interaction for egg production traits in Japanese quail. India J. Poult. Sci., 29(1): 18-22.

National Academy of Sciences (NAS)-National Research Council, 1977. Nutrient requirements of domestic animals. Nutrient requirements of poultry. $7^{\text {th }}$ Ed. NRC NAS, Washington, D.C. $62 \mathrm{pp}$

Pandey, N.K. and Shyamsunder, G. 1990. Carcass characteristics, meat yield and physicochemical properties of meat from white leghorn cockerels. Indian J. Poult. Sci., 25(4):249-252.

Shim, K.F. and Vohra, P. 1984. A review of the nutrition of Japanese quail. World's Poultry Science Journal, 40: 261-274.

Shrivastav, A.K. and Panda, B. 1982. Effect of increasing calorie-protein ratio during growing period (4-5 week) on dressing yield and chemical composition of quail meat. India J. Poult. Sci., 17: 253-256.

Singh, P.K., Khatta, V.K., Thakur, R.S., Dey, S. and Sangwan, M.L. 2003. Effects of phytase supplementation on the performance of broiler chickens fed maize and wheat based diets with different levels of non-phytate phosphorus. Asian Australas. J. Anim. Sci., 16(1):1642-1649.

Snedecor G. W. and W.G. Cochran.1989. Statistical Methods, 8th edn. The Iowa State University Press, Ames, IA, USA.

SPSS (Statistical Procedure for Social Sciences), 1998. SPSS 11.5 for windows.

Wilson, W.O., Abbott, U.K. and Abplanalp, H. 1959. Developmental and physiological studies with a new pilot animal for poultry. Coturnix quail. Poult. Sci., 38: 1260. 
Woodard, A.E., Abplanalp, H., Wilson, W.O. and Vohra, P. 1973. Japanese quail husbandry in the laboratory (Coturnix coturnix japonica). Department of Avian Sciences, University of California, Davis, 22 pp. 